Access to this work was provided by the University of Maryland, Baltimore County (UMBC)

ScholarWorks@UMBC digital repository on the Maryland Shared Open Access (MD-SOAR) platform.

Please provide feedback

Please support the ScholarWorks@UMBC repository by emailing scholarworks-group@umbc.edu and telling us what having access to this work means to you and why it's important to you. Thank you. 


\title{
Generation and Detection of Gap-Free Broadband Terahertz Radiation Using Poled Polymer Films
}

\author{
Xuemei Zheng, Alexander M. Sinyukov, and L. Michael Hayden \\ Department of Physics, University of Maryland, Baltimore County, Maryland 21250, USA \\ xzheng@umbc.edu
}

\begin{abstract}
We present a terahertz generation and detection system using poled electro-optic polymer films as the terahertz emitter and sensor, respectively. The system provides $>10 \mathrm{THz}$ gap-free spectrum band. (C)2005 Optical Society of America

OCIS codes: (320.7100) Ultrafast nonlinear optics; (250.2080) Electro-optic polymers
\end{abstract}

The last two decades have seen a great advance in terahertz (THz) technology and science. The relatively recent accessible THz electromagnetic spectral band has been widely employed in fundamental materials $[1,2,3]$, medical and biological studies [4]. Space and defense industries can also benefit from the advance of this technology. The existing and promising $\mathrm{THz}$ applications have brought on challenges for both $\mathrm{THz}$ emitters and sensors. For $\mathrm{THz}$ emitters, efficient and broadband $\mathrm{THz}$ radiation is desired; and for $\mathrm{THz}$ sensors, sensitivity and broad frequency response is critical. While using semiconductor photoconductive elements as both the THz emitters and the sensors has the advantage of great emission efficiency and detection sensitivity, achievable bandwidth without postexperimental data reconstruction from this technique can be up to only a few $\mathrm{THz}$, which is limited by the photoconductor carrier lifetime [1]. On the other hand, THz emission by the optical rectification effect of electrooptic (EO) materials and $\mathrm{THz}$ detection by free-space $\mathrm{EO}$ sampling has proven to be effective for obtaining ultrabroadband THz field. The key parts of this all-optical THz technology are to select/make suitable EO materials and to employ ultra-short laser pulse sources.

Frequency response studies of EO materials have shown that several factors can affect the bandwidth of the detected THz radiation $[5,6,7,8]$. The first factor is the laser bandwidth that interacts with the EO materials. For example, with a commercially available femtosecond laser that provides 30-nm FWHM bandwidth centered at 800 $\mathrm{nm}$, the corresponding FWHM bandwidth in the frequency domain is $\sim 14 \mathrm{THz}$, considering the relationship: $|\Delta f|=\frac{c}{\lambda_{0}{ }^{2}}|\Delta \lambda|$. If all the frequency components of the laser pulse and the corresponding $\mathrm{THz}$ radiation via the difference-frequency mixing are perfectly phase matched in the optical rectification process inside the EO material, then the best FWHM bandwidth of the THz emission will be limited up to $\sim 14 \mathrm{THz}$. The second factor is dispersion of the nonlinear coefficients of the materials. This dispersion is strong around the Reststrahlen band of EO crystals. The third factor is dispersion of the complex refractive index $\tilde{n}(\omega)$, which is also closely related to the Restrahlen band in the case of EO crystals. The dispersion of the real part of $\tilde{n}(\omega)$ results in a frequency dependent coherence length, or, velocity mismatch; and the dispersion of the imaginary part of $\tilde{n}(\omega)$ results in the strong absorption around the Reststrahlen band. In order to experimentally achieve an ultra-broadband $\mathrm{THz}$ signal, it should also be kept in mind that the optical probing pulse-width must be narrow enough to effectively sample the broadband $\mathrm{THz}$ radiation associated with the ultra-short electric field oscillation period.

One of the advantages of using amorphous EO polymers as $\mathrm{THz}$ emitters and sensors is that there is no Reststrahlen band, so that the dispersion effect does not affect the $\mathrm{THz}$ bandwidth as bad as in EO crystals where lattice resonance effect causes strong dispersion and absorption. In this paper, we present a gap-free broadband $\mathrm{THz}$ system using an EO polymer emitter and sensor.

The EO polymer films we work with are composites of 40\% DCDHF-6-V/60\% APC (DAPC). The preparation of our materials has been described elsewhere [8,9]. Briefly, films are cast from solution onto indium tin oxide coated glass substrates. After solvent evaporation, two solid polymer films are pressed in vacuum $70{ }^{\circ} \mathrm{C}$ above its glass transition temperature $T_{g}$ for $10 \sim 15$ minutes. The thickness of the resulting film is controlled by appropriate polyimide spacers. Films with thickness in the range of 50-350 $\mu \mathrm{m}$ can be obtained with this method. For the particular experiment to be presented in this paper, we use a 70- $\mu \mathrm{m}$-thick DAPC film as the THz emitter and a 130- 
$\mu \mathrm{m}$-thick DAPC film as the THz sensor. Both films have been poled across the layer with high electric field $(\sim 90$ $\mathrm{V} / \mu \mathrm{m})$ while being heated up to $T_{g}$ to achieve the polar order, essential for the high $2^{\text {nd }}$-order nonlinear optical susceptibility. The films are then cooled down to the room temperature, with the external poling voltage still applied, to freeze in the orientation of the chromophores. After fabricating the films sandwiches, we use ellipsometric technique to measure the EO coefficients of the films [10]. The 70- $\mu$ m-thick DAPC film exhibits $r_{33} \approx 42 \mathrm{pm} / \mathrm{V}$ and the $130-\mu \mathrm{m}$-thick DAPC film exhibits $r_{33} \approx 50 \mathrm{pm} / \mathrm{V}$ at $800 \mathrm{~nm}$. We do not observe significant degradation of the $\mathrm{THz}$ performance of the EO polymer films over a few weeks.

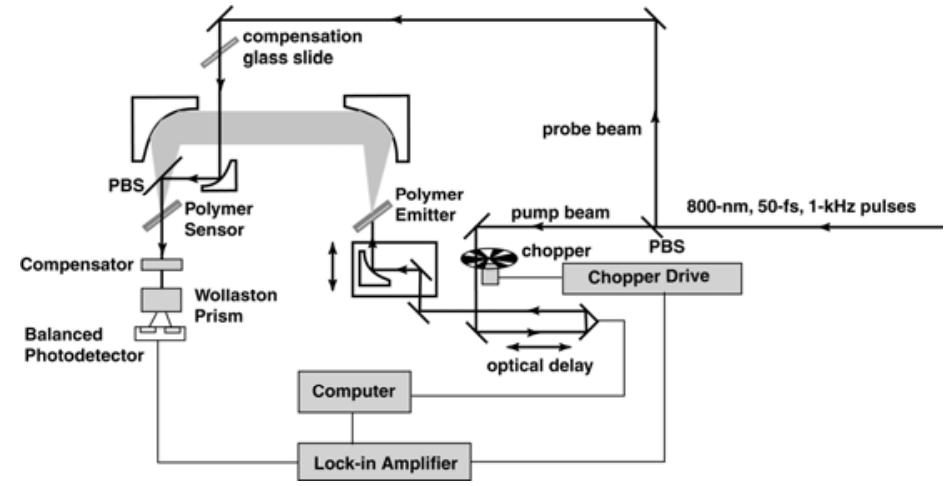

Fig. 1. The schematic of the THz system with polymer emitter and sensor.

The heart of our THz system is a regenerative laser pulse amplifier (Spectra Physics Spitfire) that provides $~ 30$ $\mathrm{nm}$ bandwidth centered at $800 \mathrm{~nm}$ and $\sim 50$-fs laser pulse duration. While using a standard $\mathrm{THz}$ generation and detection scheme for our system, we carefully design the system such that both the pump beam leg and probe beam leg go through the same amount of dispersive media, which makes group velocity dispersion (GVD) compensation possible to achieve the shortest pulse duration for both legs by adjusting the grating-pair compressor of the Spitfire. Figure 1 shows the schematic of our experimental set-up. The laser beam from the Spitfire, containing a pulse train (32 $\mu \mathrm{J} /$ pulse, $1 \mathrm{kHz}$ ), is split into pump and probe beams by a pellicle beam splitter (PBS). The pump beam is modulated by an optical chopper and goes through a computer controlled delay line. The chopped pump beam is then focused by a parabolic mirror (with effective focal length of $50.8 \mathrm{~mm}$ ) and made incident on the DAPC polymer film, with the average pump power of $\sim 10 \mathrm{~mW}$. In order to achieve maximum $\mathrm{THz}$ emission from the polymer, with a $p$-polarized pump beam, we orient the polymer film such that the pump beam is incident at Brewster's angle. The incidence is from the side of the glass substrate to avoid $\mathrm{THz}$ emission being absorbed by the glass and ITO coating. However, this causes some incident pulse-width broadening, which needs to be compensated by adjusting the grating-pair compressor in the amplifier. Keeping in mind that the probe beam pulse-width should also be as short as possible, we insert an identical glass slide in the probe beam pathway, also at Brewster's angle. In this way, both the pump and probe pulses are compressed to their shortest pulse duration. The generated THz field is collimated and focused onto the DAPC polymer sensor by a pair of off-axis parabolic mirrors. Because the DAPC films are poled normal to the substrate, and the generated THz field is $p$-polarized, the DAPC film sensor is rotated $45^{\circ}$ with respect to the $p$-polarized $\mathrm{THz}$ field, providing a projected component of the $\mathrm{THz}$ field along the poling direction of the DAPC polymer. This overlap is essential for EO detection. For the best EO detection using an EO polymer sensor, the polarization of the probe beam should be kept at $45^{\circ}$ with regard to the incidence plane. However, insertion of any half-wave plate to rotate the probe beam polarization will broaden our 50 -fs laser pulsewidth. Therefore, alternatively, we further rotate the DAPC film $45^{\circ}$ with respect to the probe beam polarization plane. A conventional balanced detection scheme is employed to achieve a good signal-to-noise ratio.

The left side of Fig. 2 shows one typical spectrum of our THz system and the right side shows the corresponding time-domain $\mathrm{THz}$ field. The spectrum is continuous with observable frequency components up to $>10 \mathrm{THz}$. Some water vapor absorption lines can be seen due to the not very dry experimental environment. Our result is very different from those obtained from such EO crystals as ZnTe [11], GaAs [12], GaP [7] and DAST [13]. By using extremely thin EO crystals, the phase mismatching problem can be overcome to some degree to obtain $\mathrm{THz}$ bandwidths as high as $37 \mathrm{THz}$ [11], but lattice resonance induced $\mathrm{THz}$ absorption can never be ignored and this phonon absorption effect always results in gaps in the ultra-broadband $\mathrm{THz}$ spectrum obtained from these $\mathrm{EO}$ 
crystals. For example, ZnTe has strong absorption around $5.3 \mathrm{THz}$, GaAs from 7 to $10 \mathrm{THz}, \mathrm{GaP}$ around $11 \mathrm{THz}$, and DAST has several strong absorption bands below $2.5 \mathrm{THz}$ [14]. Another disadvantage of using extremely thin EO crystals to obtain ultra-broadband $\mathrm{THz}$ field is the fabrication challenge, since cutting, polishing and mounting thin crystals can be very difficult.
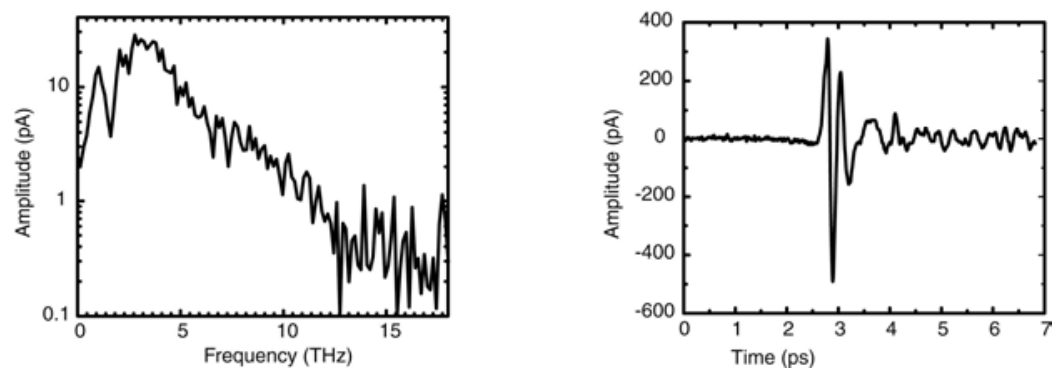

Fig. 2. The THz field emitted from a 70- $\mu \mathrm{m}$ DAPC film and detected by a $130-\mu \mathrm{m}$ DAPC film. The spectrum of the THz field by Fourier transform (left), and the corresponding time-domain trace (right).

There are two reasons, we believe, that can explain the gap-free broadband $\mathrm{THz}$ spectrum from our polymer $\mathrm{THz}$ emitter and sensor system. First of all, there is no lattice resonance region existing for our amorphous polymer films, so there is no phonon absorption in the THz regime. Second of all, the phase mismatching in the polymer films is much smaller than EO crystals [12], so the first spectrum dip due to the phase mismatching is well above $10 \mathrm{THz}$. By reducing the thickness of the polymer film, this number can be pushed to higher value. It is even possible to engineer the refractive index of polymer materials to achieve almost perfect phase matching.

Due to the gain narrowing effect, the amplifier can provide only up to $30 \mathrm{~nm}$ emission bandwidth. By using a sub-15-fs oscillator that provides $>60 \mathrm{~nm}$ emission bandwidth, we believe, the achievable $\mathrm{THz}$ radiation bandwidth can be much broadened. However, with such short laser pulse duration, both the second order and third order GVD have to be taken into consideration to obtain the shortest laser pulse-width. Unlike the second order GVD, the third order GVD can only be reduced by making the pulses pass through as short as possible dispersive media. For this reason, the substrates of the polymer films should be removed after the fabrication to obtain free-standing films, and the compensation glass slide in the probe beam pathway should also be removed accordingly. For the same reason, the polymer films should be thin to minimize the pulse broadening effect, yet it should be thick enough to have strong $\mathrm{THz}$ emission and achieve sensitive $\mathrm{THz}$ detection. We continue to work on these issues to further improve the bandwidth of our THz system.

\section{References:}

1. D. Grischkowsky, S. Keiding, M. van Exter, and Ch. Fattiger, "Far-infrared time-domain spectroscopy with terahertz beams of dielectrics and semiconductors," J. Opt. soc. Am. B 7, 2006-2015 (1990).

2. R. Huber, F. Tauser, A. Brodschelm, M. Bichler, G. Abstreiter, and A. Leitenstorfer, "How many-particle interactions develop after ultrafast excitation of an electron-hole plasma," Nature 414, 286-289 (2001).

3. R. A. Kaindl, M. A. Carnahan, J. Orenstein, D. S. Chemla, H. M. Christen, H. -Y. Zhai, M. Paranthaman, and D. H. Lowndes, "Farinfrared optical photoconductivity gap in superconducting $\mathrm{MgB}_{2}$ films,” Phys. Rev. Lett. 88, 027003 (2002).

4. A. G. Markelz, A. Roitberg, and E. J. Heilweil, "Pulsed terahertz spectroscopy of DNA, bovine serum albumin and collagen between 0.1 and $2.0 \mathrm{THz}, "$ Chem. Phys. Lett. 320, $42-48$ (2000).

5. H. J. Bakker, G. C. Cho, H. Kurz, Q. Wu, and X. -C. Zhang, "Distortion of terahertz pulses in electro-optic sampling," J. Opt. Soc. Am. B 15, 1795-1801 (1998).

6. G. Gallot and D. Grischkowsky, "Electro-optic detection of terahertz radiation,” J. Opt. Soc. Am. B 16, 1204-1212 (1998).

7. A. Leitenstorfer, S. Hunsche, J. Shah, M. C. Nuss and W. H. Knox, "Detectors and sources for ultrabroadband electro-optic sampling: experiment and theory," Appl. Phys. Lett. 74, 1516-1518 (1999).

8. A. M. Sinyukov, and L. M. Hayden, "Efficient electro-optic polymers for THz applications," J. Phys. Chem. B 108, 8515-8522 (2004).

9. A. M. Sinyukov and L. Michael Hayden, "Generation and detection of terahertz radiation in multi-layered electro-optic polymer films," Opt. Lett. 27, 55-57 (2002).

10. S. H. Han, and J. W. Wu, "Single-beam polarization interferometry measurement of the linear electro-optic effect in poled polymer films with a reflection configuration,” J. Opt. Soc. Am. B 14, 1131-1137 (1997).

11. Q. Wu, and X. -C. Zhang, "Free-space electro-optics sampling of mid-infrared pulses," Appl. Phys. Lett. 71, 1285-1287 (1997).

12. H. Cao, R. F. Heinz, and A. Nahata, "Electro-optic detection of femtosecond electromagnetic pulses by use of poled polymers," Appl. Phys. Lett. 27, 775-777 (2002).

13. P. F. Han, M. Tani, F. Pan, and X. -C. Zhang, "Use of the organic crystal DAST for terahertz applications," Opt. Lett. 25, 675-577 (2000).

14. M. Walther, K. Jensby, S. R. Keiding, H. Takahashi, and H. Ito, "Far-infrared properties of DAST," Opt. Lett. 25, 911-913 (2000). 